\title{
Identification of molecular characteristics induced by radiotherapy in rectal cancer based on microarray data
}

\author{
CHANG GE $^{1}$, MENGXIA WU ${ }^{1}$, GUIFANG CHEN ${ }^{1}$, GUANYING YU², DEHUI JI ${ }^{2}$ and SHAOZHAO WANG ${ }^{1}$ \\ Departments of ${ }^{1}$ Anorectal Surgery and ${ }^{2}$ Gastrointestinal Surgery, Jinan Central Hospital Affiliated to Shandong University, \\ Jinan, Shandong 250013, P.R. China
}

Received July 27, 2015; Accepted December 6, 2016

DOI: $10.3892 / \mathrm{ol} .2017 .5750$

\begin{abstract}
The present study aimed to reveal the molecular characteristics induced by radiotherapy in rectal cancer at the transcriptome level. Microarray data (ID, GSE26027) downloaded from the Gene Expression Omnibus database were re-analyzed to identify differentially expressed genes (DEGs) between rectal cancer tissues during and prior to radiotherapy. The DEGs were then inputted into the database for annotation, visualization and integrated discovery, an online tool to perform enrichment analyses, and into the search tool for the retrieval of interacting genes/proteins database to identify protein-protein interactions (PPIs). Subsequently, a PPI network was constructed, which was screened for densely connected modules. Furthermore, protein domain enrichment analysis was performed. In total, 690 DEGs, including 179 upregulated and 511 downregulated DEGs, were found in rectal cancer tissues during and prior to radiotherapy. The upregulated DEGs were significantly enriched in 'positive regulation of transport' and 'regulation of cardiac muscle contraction', while the downregulated DEGs were most markedly enriched in 'cell migration', 'cell-cell signaling', 'extracellular matrix organization' and 'blood vessel development', including prostaglandin-endoperoxide synthase 2 , transforming growth factor $\beta$-induced, $68 \mathrm{kDa}$ endothelin receptor type A, brain-derived neurotrophic factor, TIMP metallopeptidase inhibitor 1, and serpin family $\mathrm{E}$ member 1, which were the top 6 hub nodes in the PPI network. Furthermore, 2 protein domains were significantly enriched by PPI modules, including: The collagen triple helix repeat (CTHR) family members collagen type (COL) 5A2, COL9A3, COL6A3, COL21A1, COL5A3, COL11A1, COL7A1 and CTHR-containing-1; and the olfactory receptor family (OR) members OR7E24, OR7A17, OR6A2, OR1F1, OR10H3 and
\end{abstract}

Correspondence to: Dr Chang Ge, Department of Anorectal Surgery, Jinan Central Hospital Affiliated to Shandong University, 105 Jiefang Road, Lixia, Jinan, Shandong 250013, P.R. China E-mail: gechang@126.com

Key words: rectal cancer, radiotherapy, differentially expressed genes, enrichment analyses, protein-protein interaction, module
OR7A10. A total of 7 upregulated DEGs were characterized as tumor suppressor genes, and 8 downregulated DEGs were characterized as oncogenes. The biological processes or protein domains enriched by upregulated or downregulated DEGs may improve the understanding of molecular characteristics in response to radiotherapy.

\section{Introduction}

In $2014 \sim 40,000$ new cases of rectal cancer were diagnosed in the USA, with an incidence of 12.3/100,000 ranking as the second leading cause of cancer-associated mortality (1). Up to $40 \%$ patients with rectal cancer develop metastatic rectal cancer (2). The management of rectal cancer has developed into an integrated approach involving surgery, chemotherapy, radiotherapy and biological therapy (3), which has improved the 5-year survival rate of patients with rectal cancer (4).

Radiotherapy is commonly used as an adjuvant therapy for treating rectal cancer (5). Preoperative radiotherapy has been suggested as an essential aspect of the treatment options for rectal cancer, and can effectively reduce the local recurrence rate of rectal cancer (6). A number of molecular markers, including p53, p27, p21, epidermal growth factor receptor, B-cell lymphoma 2 ( $\mathrm{Bcl}-2) / \mathrm{Bcl}-2$-associated $\mathrm{X}$ protein and human phosphatidylethanolamine-binding protein 4 have been revealed to participate in the rectal cancer response to radiotherapy (7-9). However, despite the potential of radiotherapy in terms of treating rectal cancer, the treatment may cause severe side effects such as bowel dysfunction and obstruction, incontinence, sexual dysfunction, unspecified infection, abdominal pain, nausea and secondary cancer $(6,10,11)$. Thus, a comprehensive evaluation of radiotherapy for the treatment of rectal cancer is required in order to develop effective therapeutic approaches for patients with rectal cancer. Previously, transcriptomic changes in response to radiotherapy in patients with rectal cancer using microarray data were revealed (12). However, only differential expression analysis and function analysis were performed in the aforementioned study, and the microarray data can be investigated reveal the molecular response to radiotherapy. The present study re-analyzed the gene expression profiles (12) of rectal cancer during and prior to radiotherapy to screen out differentially expressed genes (DEGs) with thresholds of the absolute value of $\log _{2}$ fold change $(\mathrm{FC})>1$ and $\mathrm{P}<0.05$ which were different compared 
Table I. Top 5 GO terms and KEGG pathways significantly enriched by upregulated and downregulated DEGs.

Term P-value Count Partial gene list

DEGS upregulated

GO_BP 0051050: Positive regulation of transport

GO_BP 0055117: Regulation of cardiac muscle

contraction

GO_BP 0071276: Cellular response to cadmium ion

GO_BP 0014721: Twitch skeletal muscle contraction

GO_BP 0001508: Regulation of action potential

KEGG 4020: Calcium signaling pathway

KEGG 4972: Pancreatic secretion

KEGG 920: Sulfur metabolism

KEGG 2010: ABC transporters

DEGS downregulated

GO_BP 0016477: Cell migration

GO_BP 0007267: Cell-cell signaling

GO_BP 0030198: Extracellular matrix organization

GO_BP 0001568: Blood vessel development

GO_BP 0044243: Multicellular organismal catabolic process

KEGG 4060: Cytokine-cytokine receptor interaction

KEGG 5323: Rheumatoid arthritis

KEGG 260: Glycine, serine and threonine metabolism

KEGG 670: One carbon pool by folate

$\begin{array}{lrll}2.06^{\mathrm{E}-05} & 14 & \text { KCNQ1, NEDD4L, P2RX4, RYR2, CHRM1 } \\ 8.27^{\mathrm{E}-05} & 5 & \text { KCNQ1, NEDD4L, P2RX4, RYR2, BMP10 } \\ & & \\ 1.25^{\mathrm{E}-04} & 3 & \text { GSN, MT1E, CYP11A1 } \\ 1.54^{\mathrm{E}-04} & 2 & \text { ATP2A1, MB } \\ 1.76^{\mathrm{E}-04} & 7 & \text { KCNQ1, NEDD4L, RYR2, SLC9A1, RXRG, } \\ & & \text { EPB41L3, PLP1 } \\ 1.83^{\mathrm{E}-03} & 7 & \text { ATP2A1, P2RX4, RYR2, GNA14, ATP2A3, } \\ & & \text { CHRM1, CHP2 } \\ 3.28^{\mathrm{E}-03} & 5 & \text { KCNQ1, ATP2A1, RYR2, ATP2A3, SLC9A1 } \\ 7.22^{\mathrm{E}-03} & 2 & \text { BPNT1, SULT1E1 } \\ 9.54^{\mathrm{E}-03} & 3 & \text { ABCG1, ABCA5, ABCC12 } \\ & & \\ 1.44^{\mathrm{E}-09} & 49 & \text { PTGS2, EDNRA, TIMP1, MMP10, SERPINE1 } \\ 1.31^{\mathrm{E}-07} & 52 & \text { PTGS2, BDNF, NPVF, MPZ, BSN } \\ 4.44^{\mathrm{E}-07} & 23 & \text { TGFBI, MMP10, TIMP1, SERPINE1, COL9A3 } \\ 9.89^{\mathrm{E}-07} & 30 & \text { PTGS2, TGFBI, EDNRA, ESM1, SERPINE1 } \\ 1.24^{\mathrm{E}-06} & 11 & \text { COL5A2, MMP10, COL9A3, COL11A1, } \\ 4.85^{\mathrm{E}-05} & 17 & \text { COL5A3 } \\ 3.37^{\mathrm{E}-03} & 7 & \text { MMP1, CXCL3, CXCL1, FIGF, TNFRSF10C } \\ 4.70^{\mathrm{E}-03} & 4 & \text { PSAT1, PSPH, SHMT2, PIPOX } \\ 6.36^{\mathrm{E}-3} & 3 & \text { ALDH1L2, MTHFD1L, SHMT2 } \\ & & \end{array}$

GO, gene ontology; KEGG, Kyoto Encyclopedia of Genes and Genomes; DEGs, differentially expressed genes; Count, the number of DEGs enriched in the corresponding term; BP, biological process.

with the criteria of FC (particularly the ratio 'gene expression during radiotherapy/gene expression prior to radiotherapy') $>2.5$ or $<0.4$ and false discovery rate $<0.11$. In addition, enrichment analyses and functional annotation were subsequently performed for DEGs to reveal their biological relevance. Furthermore, protein-protein interactions (PPIs) were analyzed and visualized into a PPI network to identify hub nodes, and the modules were mined from the PPI network.

\section{Materials and methods}

Microarray data. The gene expression profiles (ID, GSE26027) (12) were obtained from the Gene Expression Omnibus database (National Institutes of Health, Bethesda, MD, USA), which were previously produced based on the platform of Affymetrix Human Genome U133 Plus 2.0 Array (Affymetrix, Inc., Santa Clara, CA, USA). The biopsies for the microarray analysis were obtained from 6 patients with locally advanced rectal cancer prior to radiotherapy and $1 \mathrm{~h}$ subsequent to a dose of 7.2 Gy by the 4 th fraction (1.8 Gy/fraction) during radiotherapy. All patients enrolled in the experiment provided written informed consent approved by the University of Nantes Institutional Review Board for human studies (Nantes, France) (12).
Identification ofDEGs. Raw expression datawere pre-processed by conducting the following analyses: Background correction; quantile normalization; probe summarization; and generation of the gene expression matrix. In order to take a global view on transcriptome changes, DEGs between biopsies taken during and prior to radiotherapy were screened out using thresholds of the absolute value of $\log _{2} \mathrm{FC}>1$ and $\mathrm{P}<0.05$ using the $t$ test implemented in Linear Models for Microarray Analysis, (Version 3.0, http://www.bioconductor.org/packages/release/ bioc/html/limma.html) package $(13,14)$.

Enrichment analyses. To identify biological functions involving DEGs, the identified DEGs were submitted to the Database for Annotation, Visualization and Integrated Discovery (DAVID), version 6.7 (15), to identify Gene Ontology (http:// geneontology.org/) functions $(16,17)$ and Kyoto Encyclopedia of Genes and Genomes (http://www.genome.jp/kegg/) pathways (18). $\mathrm{P}<0.01$ was set as the cut-off criterion.

Functional annotation. Transciption factors were identified among DEGs using the TRANSFAC database (version 10.4, http://www.gene-regulation.com/pub/databases.html), which records eukaryotic transcription factors (TFs), their binding sites and DNA binding profiles (19). Oncogenes and 
Table II. Functional annotation of upregulated and downregulated DEGs.

Upregulated DEGs

6

TF count

TF gene

INSM1, HOXA13, ATOH1, RXRG, HSF4, RORC

Oncogene count

1

Oncogene

MOS

TSG count

TSG
7

EPB41L3, CABLES1, ZBTB7C, TXNIP, BMP10, ACCN1, PDCD4
Downregulated DEGs

20

HOXC8, EGR1, GLIS2, PDX1, TAL2, FOXC1, CDX4, FOXL1, SOX17, NKX2-8, SHOX, TWIST1, TBX5, ALX3, MYF6, KLF1, POU4F2, SIX6, OLIG1, HESX1

8

AXL, PVT1, WISP1, THPO, FOSL1, MRAS, TWIST1, CXCL1

EGR1, GAS1, GJA1, DKK3, PDX1, NRSN2, PHLDA3, NRCAM, HTRA1, PLK2, LOX, FOXC1, TBX5, TNFRSF10B, RHOBTB2, PRODH, WNT5A, TGFBI, EDA2R, LSAMP, NDRG4, LPL, S100A2, IL24

DEGs, differentially expressed genes; TF, transcription factor; TSG, tumor suppressor gene; Count, the number of DEGs.

tumor suppressor genes (TSGs) were determined according to the TSGene database (20) and Tumor Associated Gene database $(21,22)$.

PPI network construction and module mining. As the interactions between proteins are important for biological metabolism, the DEGs were inputted into the Search Tool for the Retrieval of Interacting Genes (STRING) version 9.1 (23). The interaction pairs were screened from the experimental data, textual mining results, co-expression analysis findings and other interacting records in the STRING database. The interaction pairs were then visualized by constructing a PPI network via Cytoscape version 3.0 (24). Hub nodes, specifically, hub DEGs, were subsequently identified in the PPI network.

Furthermore, densely connected modules were identified from the PPI network, using the threshold of $\mathrm{P}<0.001$ and the plugin Clustering with Overlapping Neighborhood Expansion (25) in Cytoscape software.

Protein domain enrichment analysis. The InterPro database (EMBL-EBI, Hinxton, UK) provides family and domain information for the functional analysis of protein sequences (26). Based on the InterPro database, protein domain enrichment analysis was conducted in the present study for the densely connected modules using DAVID (15), classifying proteins into families and predicting their domains and important sites.

\section{Results}

DEGs. With the cut-offs of the absolute value of $\log _{2} \mathrm{FC}>1$ and $\mathrm{P}<0.05$, the present study identified 690 DEGs in rectal cancer tissues during radiotherapy compared to those prior to radiotherapy, including 179 upregulated and 511 downregulated DEGs. There were a greater number of downregulated DEGs than upregulated DEGs, indicating a tendency to downregulate in response to radiotherapy.
Results of enrichment analyses. By inputting the DEGs into the online tool DAVID, the upregulated DEGs were significantly enriched in 'positive regulation of transport' $\left(\mathrm{P}=2.06^{\mathrm{E}-05}\right)$ and 'regulation of cardiac muscle contraction' $\left(\mathrm{P}=8.27^{\mathrm{E}-05}\right)$ (Table I). However, the downregulated DEGs were most significantly enriched in 'cell migration' $\left(\mathrm{P}=1.44^{\mathrm{E}-09}\right)$ : Prostaglandin-endoperoxide synthase 2 (PTGS2), endothelin receptor type A (EDNRA), TIMP metallopeptidase inhibitor 1 (TIMP 1) and serpin family E member 1 (SERPINE 1), 'cell-cell signaling': PTGS2 and brain-derived neurotrophic factor (BDNF), 'extracellular matrix organization': Transforming growth factor $\beta$-induced, $68 \mathrm{kDa}$ (TGFBI), TIMP1 and SERPINE1, and 'blood vessel development': PTGS2, TGFBI, EDNRA and SERPINE1 (Table I).

Results of functional annotation. In total, 7 upregulated DEGs such as bone morphogenetic protein 10 (BMP10) and 8 downregulated DEGs such as twist family BHLH transcription factor 1 (TWIST1) were, respectively, identified as tumor suppressor genes and oncogenes (Table II). In addition, 6 upregulated DEGs and 20 downregulated DEGs such as TWIST1 were revealed to be TFs (Table II). However, 24 downregulated DEGs were characterized as TSGs, and 1 upregulated gene, Moloney sarcoma oncogene, was identified as an oncogene (Table II).

PPI network and modules. The interaction pairs of DEGs were inputted into Cytoscape and a PPI network was obtained containing 271 nodes and 458 interaction pairs (Fig. 1). The top 6 hub DEGs with a high degree score were identified, including PTGS2 (degree=19), TGFBI (degree=19), EDNRA (degree=14), BDNF (degree=13), TIMP1 (degree=13) and SERPINE1 (degree=13).

Furthermore, using the threshold $\mathrm{P}<0.01$, a total of 3 modules were mined from the PPI network, including modules $1\left(\mathrm{P}=7.42^{\mathrm{E}-05}\right), 2\left(\mathrm{P}=1.37^{\mathrm{E}-04}\right)$ and $3\left(\mathrm{P}=6.31^{\mathrm{E}-04}\right.$; 


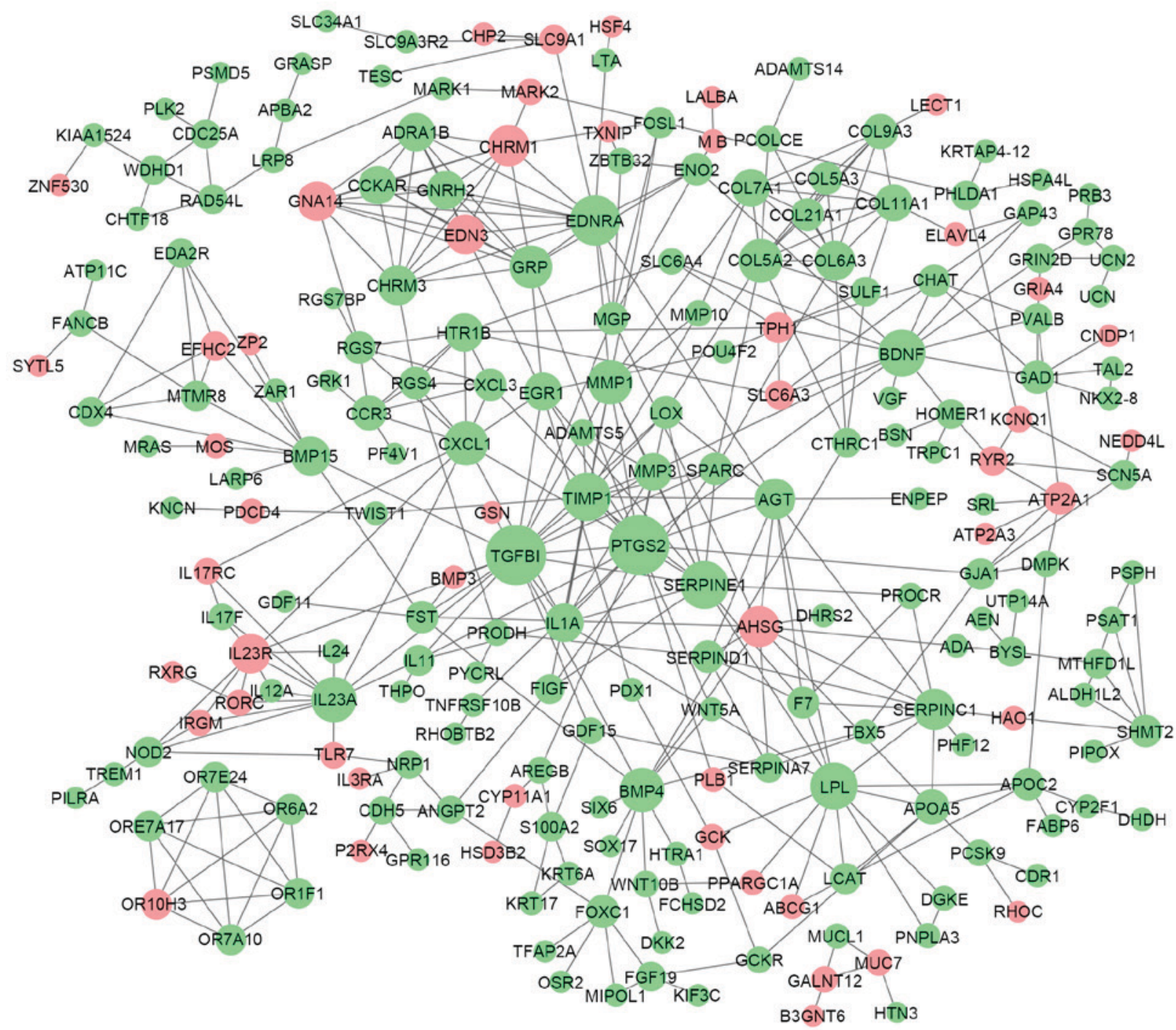

Figure 1. Protein-protein interaction network of DEGs. Red and green nodes indicate upregulated and downregulated DEGs, respectively, while lines indicate interactions between DEGs. The size of each node indicates the number of interactions with other DEGs. DEGs, differentially expressed genes.

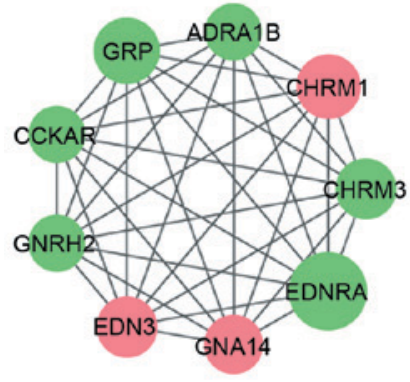

M1

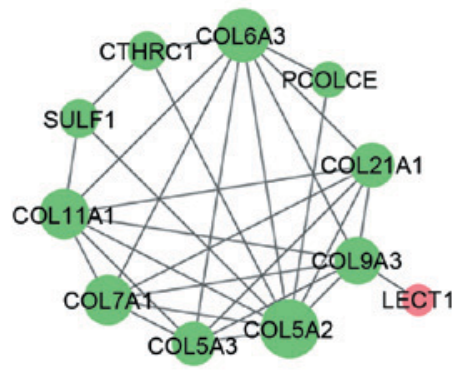

M2

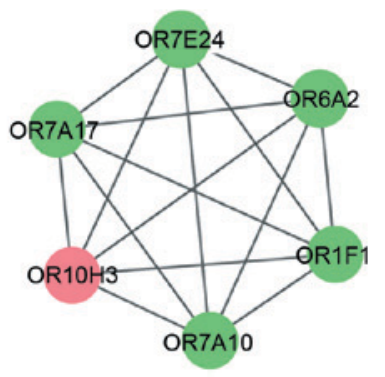

M3

Figure 2. Densely connected modules screened from the protein-protein interaction network. Red and green nodes indicate upregulated and downregulated DEGs, respectively, while lines indicate interactions between DEGs. The size of each node indicates the number of interactions with other DEGs. M1, module 1; M2, module 2; M3, module 3; DEGs, differentially expressed genes.

Fig. 2). DEGs with a high degree score in modules were found, including ENDRA in module 1 and collagen type $\mathrm{V} \alpha 2$ (COL5A2) in module 2.

Results of protein domain enrichment analysis. By performing protein domain enrichment analysis, module 2 was revealed to be most significantly enriched in the protein domain 'collagen triple helix repeat' $\left(\mathrm{P}=7.60^{\mathrm{E}-15}\right)$, e.g. COL5A2 and collagen triple helix repeat containing 1 (CTHRC1), while module 3 was markedly associated with protein domain 'olfactory receptor' $\left(\mathrm{P}=1.09^{\mathrm{E}-8}\right.$; Table III). No protein domain was enriched by the proteins in module 1 (Table III). 
Table III. Results of protein domain enrichment analysis on the differentially expressed genes.

\begin{tabular}{|c|c|c|c|}
\hline Term & P-value & Count & Gene list \\
\hline Module 1 & - & 0 & None \\
\hline \multicolumn{4}{|l|}{ Module 2} \\
\hline IPR008160: Collagen triple helix repeat & $7.60^{\mathrm{E}-15}$ & 8 & $\begin{array}{l}\text { COL5A2, COL9A3, COL6A3, COL21A1, } \\
\text { COL5A3, COL11A1, COL7A1, CTHRC1 }\end{array}$ \\
\hline IPR000885: Fibrillar collagen, C-terminal & $1.78^{\mathrm{E}-05}$ & 3 & COL5A2, COL5A3, COL11A1 \\
\hline IPR003129: Laminin G, thrombospondin-type, N-terminal & $7.44^{\mathrm{E}-05}$ & 3 & COL21A1, COL5A3, COL11A1 \\
\hline IPR002035: von Willebrand factor, type A & $9.75^{\mathrm{E}-04}$ & 3 & COL7A1, COL21A1, COL6A3 \\
\hline \multicolumn{4}{|l|}{ Module 3} \\
\hline IPR000725: Olfactory receptor & $1.09^{\mathrm{E}-08}$ & 6 & $\begin{array}{l}\text { OR7E24, OR7A17, OR6A2, OR1F1, } \\
\text { OR10H3, OR7A10 }\end{array}$ \\
\hline IPR017452: GPCR, rhodopsin-like superfamily & $1.55^{\mathrm{E}-07}$ & 6 & $\begin{array}{l}\text { OR7E24, OR7A17, OR6A2, OR1F1, } \\
\text { OR10H3, OR7A10 }\end{array}$ \\
\hline IPR000276: 7 transmembrane GPCR, rhodopsin-like & $1.56^{\mathrm{E}-07}$ & 6 & $\begin{array}{l}\text { OR7E24, OR7A17, OR6A2, OR1F1, } \\
\text { OR10H3, OR7A10 }\end{array}$ \\
\hline
\end{tabular}

GPCR, G-protein coupled receptor; COL, collagen type; OR, olfactory receptor.

\section{Discussion}

Currently, radiotherapy is an important treatment approach for rectal cancer, although the treatment may induce body damage $(10,27)$. The present study provides a comprehensive view on transcriptome changes in response to radiotherapy by re-analyzing previous microarray data (12). As a result, 179 upregulated and 511 downregulated DEGs were identified with the criteria of the absolute value of $\log _{2} \mathrm{FC}>1$ and $\mathrm{P}<0.05$. In a previous study, Supiot et al (12) found that 31 and 6 DEGs were upregulated and downregulated, respectively, by radiotherapy with the criteria of FC (specifically, the ratio 'gene expression during radiotherapy/gene expression prior to radiotherapy') $>2.5$ or $<0.4$ and false discovery rate $<0.11$. The differences of DEG numbers between the present and previous study may be due to the distinct P-value thresholds. The criteria used in the present study are commonly utilized in differential expression analysis, therefore the DEGs identified are reasonable.

Subsequent to the enrichment analysis, the upregulated DEGs were mainly associated with the regulation of transport and cardiac muscle contraction. Ion transport is important for cardiac muscle contraction (28), which can be affected by cranial radiotherapy in individuals that survived childhood cancer (29). In the present study, the DEGs involved in the aforementioned processes were significantly upregulated by radiotherapy, suggesting the effects of radiotherapy on cardiac muscle contraction. In addition, the top 4 biological processes significantly enriched by downregulated DEGs were cell migration, cell-cell signaling, extracellular matrix organization and blood vessel development. It has been reported that cell migration and cell-cell communication are important for rectal cancer progression and metastasis $(30,31)$, extracellular matrix organization and angiogenesis (32). The downregulated hub DEGs in the PPI network, including PTGS2, TGFBI, EDNRA, BDNF, TIMP1 and SERPINE1, were revealed to be involved in the aforementioned processes. The level of PTGS2 expression, which exhibits predictive usage for managing rectal cancer (33), is increased by preoperative radiotherapy and involved in local relapse (34). TGFBI serves as a linker protein, and the overexpression of the protein contributes to colorectal cancer development (35). The upregulation of BDNF, SERPINE1 and TIMP1 are associated with colorectal cancer metastasis (36-38). Therefore, the present study hypothesizes that the downregulation of the aforementioned DEGs may alter the biological processes associated with rectal cancer progression and metastasis, which were important molecular responses to radiotherapy in rectal cancer. However, the downregulation of EDNRA, a tumor suppressor, contributes to colorectal cancer progression (21). A decrease in the expression level of BDNF is associated with irradiation-induced hippocampal neurogenesis impairment in Sprague Dawley rats (39). Therefore, radiotherapy in rectal cancer may also cause side effects via regulating the aforementioned genes.

In addition, 3 densely connected modules were identified in the PPI network, and the proteins in 2 of the modules were, respectively, enriched in the protein domains 'collagen triple helix repeat', e.g. CTHRC1 and COL5A2, and 'olfactory receptor'. Collagen is a major component of the interstitial extracellular matrix, which performs a role in cellular proliferation, differentiation, apoptosis, migration and carcinogenesis (40). Due to the interaction with the collagen triple helix repeat domain, CTHRC1 promotes rectal cancer invasion and metastasis with vascular endothelial growth factor C (41), while COL5A2 is co-expressed with COL11A1 in colorectal carcinomas and associated with malignancy in colorectal cancer (40). Numerous types of collagen were also significantly enriched in this domain, and thus may be involved in extracellular matrix change in response to radiotherapy $(42,43)$. Olfactory receptors in the olfactory epithelium are usually overexpressed in tumors and promote cellular 
invasion and metastasis (44). In the present study, the olfactory receptor-associated DEGs in module 3 were downregulated by radiotherapy and thus may contribute towards preventing rectal cancer progression.

In the present study, 7 TSGs such as BMP10 were upregulated and 8 oncogenes including TWIST1 were downregulated in rectal cancer tissues subsequent to radiotherapy. BMP10 is a tumor suppressor in urothelial, prostate and breast cancer, and the overexpression of the gene inhibits the growth, adhesion, migration and invasion of cancer cells $(45,46)$. As an oncogene involved in the epithelial-to-mesenchymal transition, TWIST1 possesses angiogenic, invasive, oncogenic and drug-resistant properties in human tumors. In colorectal cancer, TWIST1 is overexpressed and correlates with lymph node metastasis, overall survival and disease-free survival rates (47). Therefore, radiotherapy may benefit patients with rectal cancer via upregulating TSGs and downregulating oncogenes. However, although 24 TSGs were downregulated, 1 oncogene was upregulated, indicating that radiotherapy in rectal cancer may also promote cancer development.

In conclusion, the present study revealed various molecular responses to radiotherapy in rectal cancer. Cardiac muscle contraction may be affected by radiotherapy in patients with rectal cancer, while metastasis-associated biological processes may be prevented by radiotherapy. In addition, enriched protein domains such as the collagen triple helix repeat and olfactory receptors suggested that radiotherapy degrades the extracellular matrix and promotes metastasis in rectal cancer. In comparison with a previous study (12) that used the dataset GSE26027, the present study identified more DEGs using reasonable criteria, found a potential hub of DEGs by constructing a PPI network and identified TFs, oncogenes and TSGs in DEGs, providing information on the effects of radiotherapy at a molecular level. However, the hypotheses of the present study were formed based on the microarray data of only 6 patients. Therefore, additional exploration and validation is required, and the present authors are planning to use more samples to verify the DEGs with a high degree score in PPI network in future studies, and the annotated TFs, oncogenes and TSGs.

\section{References}

1. Siegel R, Ma J, Zou Z and Jemal A: Cancer statistics, 2014. CA Cancer J Clin 64: 9-29, 2014

2. Glynne-Jones R and Kronfli M: Locally advanced rectal cancer: A comparison of management strategies. Drugs 71: 1153-1177, 2011.

3. Yaffee P, Osipov A, Tan C, Tuli R and Hendifar A: Review of systemic therapies for locally advanced and metastatic rectal cancer. J Gastrointest Oncol 6: 185-200, 2015.

4. Howlander N, Noone A, Krapcho M, Garshell J, Miller D and Altekruse S: SEER cancer statistics review, 1975-2011. Bethesda, MD: National Cancer Institute 2014, 2015.

5. Smith $\mathrm{N}$ and Brown G: Preoperative staging of rectal cancer. Acta Oncol 47: 20-31, 2008.

6. Birgisson H, Påhlman L, Gunnarsson U and Glimelius B; Swedish Rectal Cancer Trial Group: Adverse effects of preoperative radiation therapy for rectal cancer: Long-term follow-up of the Swedish rectal cancer trial. J Clin Oncol 23: 8697-8705, 2005.

7. Lin LC, Lee HH, Hwang WS, Li CF, Huang CT, Que J, Lin KL, Lin FC and Lu CL: p53 and p27 as predictors of clinical outcome for rectal-cancer patients receiving neoadjuvant therapy. Surg Oncol 15: 211-216, 2006.
8. Kuremsky JG, Tepper JE and McLeod HL: Biomarkers for response to neoadjuvant chemoradiation for rectal cancer. Int $\mathrm{J}$ Radiat Oncol Biol Phys 74: 673-688, 2009.

9. Qiu J, Yang G, Shen Z, Xie Y and Wang L: hPEBP4 as a predictive marker for the pathological response of rectal cancer to preoperative radiotherapy. Int J Colorectal Dis 28: 241-246, 2013.

10. Stelzmueller I, Zitt M, Aigner F, Kafka-Ritsch R, Jäger R, De Vries A, Lukas P, Eisterer W, Bonatti H and Ofner D: Postoperative morbidity following chemoradiation for locally advanced low rectal cancer. J Gastrointest Surg 13: 657-667, 2009.

11. Birgisson H, Påhlman L, Gunnarsson U and Glimelius B: Late adverse effects of radiation therapy for rectal cancer-a systematic overview. Acta Oncol 46: 504-516, 2007.

12. Supiot S, Gouraud W, Campion L, Jezéquel P, Buecher B, Charrier J, Heymann MF, Mahé MA, Rio E and Chérel M: Early dynamic transcriptomic changes during preoperative radiotherapy in patients with rectal cancer: A feasibility study. World J Gastroenterol 19: 3249-3254, 2013.

13. Wettenhall JM and Smyth GK: limmaGUI: A graphical user interface for linear modeling of microarray data. Bioinformatics 20 : 3705-3706, 2004.

14. Ritchie ME, Phipson B, Wu D, Hu Y, Law CW, Shi W and Smyth GK: limma powers differential expression analyses for RNA-sequencing and microarray studies. Nucleic Acids Res 43: e47, 2015.

15. Huang DW, Sherman BT, Tan Q, Collins JR, Alvord WG, Roayaei J, Stephens R, Baseler MW, Lane HC and Lempicki RA: The DAVID gene functional classification tool: A novel biological module-centric algorithm to functionally analyze large gene lists. Genome Biol 8: R183, 2007.

16. Ashburner M, Ball CA, Blake JA, Botstein D, Butler H, Cherry JM, Davis AP, Dolinski K, Dwight SS, Eppig JT, et al: Gene ontology: Tool for the unification of biology. The gene ontology consortium. Nat Genet 25: 25-29, 2000.

17. Gene Ontology Consortium: Gene ontology consortium: Going forward. Nucleic Acids Res 43 (Database Issue): D1049-D1056, 2015.

18. Du J, Yuan Z, Ma Z, Song J, Xie X and Chen Y: KEGG-PATH: Kyoto encyclopedia of genes and genomes-based pathway analysis using a path analysis model. Mol Biosyst 10: 2441-2447, 2014.

19. Wingender E, Dietze P, Karas H and Knüppel R: TRANSFAC: A database on transcription factors and their DNA binding sites. Nucleic Acids Res 24: 238-241, 1996.

20. Zhao M, Sun J and Zhao Z: TSGene: A web resource for tumor suppressor genes. Nucleic Acids Res 41 (Database Issue): D970-D976, 2013.

21. Chen C, Wang L, Liao Q, Huang Y, Ye H, Chen F, Xu L, Ye M and Duan S: Hypermethylation of EDNRB promoter contributes to the risk of colorectal cancer. Diagn Pathol 8: 199, 2013.

22. Chen JS, Hung WS, Chan HH, Tsai SJ and Sun HS: In silico identification of oncogenic potential of fyn-related kinase in hepatocellular carcinoma. Bioinformatics 29: 420-427, 2013.

23. von Mering C, Huynen M, Jaeggi D, Schmidt S, Bork P and Snel B: STRING: A database of predicted functional associations between proteins. Nucleic Acids Res 31: 258-261, 2003.

24. Kohl M, Wiese S and Warscheid B: Cytoscape: Software for visualization and analysis of biological networks. In: Data Mining in Proteomics Springer, pp291-303, 2011.

25. Nepusz T, Yu H and Paccanaro A: Detecting overlapping protein complexes in protein-protein interaction networks. Nat Methods 9: 471-472, 2012.

26. Hunter S, Jones P, Mitchell A, Apweiler R, Attwood TK, Bateman A, Bernard T, Binns D, Bork P, Burge S, et al: InterPro in 2011: new developments in the family and domain prediction database. Nucleic Acids Res 40 (Database Issue): D306-D312, 2012.

27. Valentini V and Glimelius B: Rectal cancer radiotherapy: Towards European consensus. Acta Oncol 49: 1206-1216, 2010.

28. Ferrantini C, Coppini R, Sacconi L, Tosi B, Zhang ML, Wang GL, de Vries E, Hoppenbrouwers E, Pavone F, Cerbai E, et al: Impact of detubulation on force and kinetics of cardiac muscle contraction. J Gen Physiol 143: 783-797, 2014

29. Hummel YM, Hooimeijer HL, Zwart N, Tissing WJ, Gietema JA, Voors AA and van den Berg MP: Long-term cardiac abnormalities after cranial radiotherapy in childhood cancer survivors. Acta Oncol 54: 515-521, 2015. 
30. Salvans S, Mayol X, Alonso S, Messeguer R, Pascual M, Mojal S, Grande L and Pera M: Postoperative peritoneal infection enhances migration and invasion capacities of tumor cells in vitro: An insight into the association between anastomotic leak and recurrence after surgery for colorectal cancer. Ann Surg 260: 939-944, 2014

31. Zhang Z and DuBois RN: Detection of differentially expressed genes in human colon carcinoma cells treated with a selective COX-2 inhibitor. Oncogene 20: 4450-4456, 2001.

32. Xu B, Shen F, Cao J and Jia L: Angiogenesis in liver metastasis of colo-rectal carcinoma. Front Biosci (Landmark Ed) 18 1435-1443, 2013.

33. Lobo Prabhu KC, Vu L, Chan SK, Phang T, Gown A, Jones SJ and Wiseman SM: Predictive utility of cyclo-oxygenase-2 expression by colon and rectal cancer. Am J Surg 207: 712-716, 2014.

34. Bouzourene H, Yan P, Sandmeier D, Zouhair A, Matter M, Vuilleumier H and Coucke P: The role of COX-2 in rectal cancer treated with preoperative radiotherapy. Virchows Arch 452: 499-505, 2008

35. Zhu J, Chen X, Liao Z, He C and Hu X: TGFBI protein high expression predicts poor prognosis in colorectal cancer patients. Int J Clin Exp Pathol 8: 702-710, 2015.

36. Tanaka K, Okugawa Y, Toiyama Y, Inoue Y, Saigusa S, Kawamura M, Araki T, Uchida K, Mohri Y and Kusunoki M: Brain-derived neurotrophic factor (BDNF)-induced tropomyosin-related kinase B (Trk B) signaling is a potential therapeutic target for peritoneal carcinomatosis arising from colorectal cancer. PLoS One 9: e96410, 2014.

37. Bunatova K, Pesta M, Kulda V, Topolcan O, Vrzalova J, Sutnar A, Treska V, Pecen L and Liska V: Plasma TIMP1 level is a prognostic factor in patients with liver metastases. Anticancer Res 32 . 4601-4606, 2012.

38. Mazzoccoli G, Pazienza V, Panza A, Valvano MR, Benegiamo G, Vinciguerra M, Andriulli A and Piepoli A: ARNTL2 and SERPINE1: Potential biomarkers for tumor aggressiveness in colorectal cancer. J Cancer Res Clin Oncol 138: 501-511, 2012.
39. Ji S, Tian Y, Lu Y, Sun R, Ji J, Zhang L and Duan S: Irradiation-induced hippocampal neurogenesis impairment is associated with epigenetic regulation of bdnf gene transcription. Brain Res 1577: 77-88, 2014.

40. Fischer H, Stenling R, Rubio C and Lindblom A: Colorectal carcinogenesis is associated with stromal expression of COL11A1 and COL5A2. Carcinogenesis 22: 875-878, 2001.

41. Yin WH, Fan HZ, Sheng JW, Xia HM, Wu YW and Xie P: Effect of vascular endothelial growth factor $\mathrm{C}$ and collagen triple helix repeat containing 1 expression on prognosis of rectal carcinoma patients. Zhonghua Wei Chang Wai Ke Za Zhi 16: 673-675, 2013 (In Chinese).

42. Guenther U, Herbst H, Bauer M, Isbert C, Buhr HJ, Riecken EO and Schuppan D: Collagen type XVIII/endostatin is differentially expressed in primary and metastatic colorectal cancers and ovarian carcinomas. Br J Cancer 85: 1540-1545, 2001.

43. Tarpey PS, Behjati S, Cooke SL, Van Loo P, Wedge DC, Pillay N, Marshall J, OMeara S, Davies H, Nik-Zainal S, et al: Frequent mutation of the major cartilage collagen gene COL2A1 in chondrosarcoma. Nat Genet 45: 923-926, 2013.

44. Sanz G, Leray I, Dewaele A, Sobilo J, Lerondel S, Bouet S, Grébert D, Monnerie R, Pajot-Augy E and Mir LM: Promotion of cancer cell invasiveness and metastasis emergence caused by olfactory receptor stimulation. PLoS One 9: e85110, 2014.

45. Zhang N, Ye L, Wu L, Deng X, Yang Y and Jiang WG: Expression of bone morphogenetic protein-10 (BMP10) in human urothelial cancer of the bladder and its effects on the aggressiveness of bladder cancer cells in vitro. Anticancer Res 33: 1917-1925, 2013.

46. Ye L, Bokobza S, Li J, Moazzam M, Chen J, Mansel RE and Jiang WG: Bone morphogenetic protein-10 (BMP-10) inhibits aggressiveness of breast cancer cells and correlates with poor prognosis in breast cancer. Cancer Sci 101: 2137-2144, 2010.

47. Gomez I, Peña C, Herrera M, Muñoz C, Larriba MJ, Garcia V, Dominguez G, Silva J, Rodriguez R, Garcia de Herreros A, et al: TWIST1 is expressed in colorectal carcinomas and predicts patient survival. PLoS One 6: e18023, 2011. 